Pensar en Movimiento:

Revista de Ciencias del Ejercicio y la Salud

EISSN 1659-4436

Vol. 13, No.1, pp. $1-4$

\title{
NUEVAS FORMAS DE REVISIÓN POR PARES EN REVISTAS CIENTÍFICAS: REVISIÓN ABIERTA /OPEN REVIEW
}

\author{
NEW WAYS OF PEER REVIEWING IN JOURNALS: OPEN REVIEW
}

\author{
Andrea Mora Campos, M. Sc. \\ andrea.moracampos@ucr.ac.cr \\ Escuela de Educación Física y Deportes, Universidad de Costa Rica, Costa Rica \\ Manuscrito publicado: 30/06/2015 \\ doi: http://dx.doi.org/10.15517/pensarmov.v13i1.19942
}

\begin{abstract}
RESUMEN
Mora-Campos, A. (2015). Nuevas formas de revisión por pares en revistas científicas: revisión abierta lopen review. PENSAR EN MOVIMIENTO: Revista de Ciencias del Ejercicio y la Salud, 13(1), 1-4. La tecnología ha ayudado a mejorar muchos aspectos de la calidad de las publicaciones: más visibilidad, mayor acceso a las fuentes de información y opción de verificar fuentes, entre otros. Esto ha implicado que algunas revistas tengamos que ajustar procesos, en especial en la de revisión por pares. El sistema más utilizado por las revistas es la modalidad ciego. En el caso de nuestra revista, tradicionalmente hemos utilizado el estilo de doble ciego, pero nos hemos dado cuenta que con el avance del Internet, las redes sociales, o los medios de difusión es posible que muchos de los dictaminadores lleguen a obtener previos, preprint, investigaciones primarias o bien artículos anteriores que facilitan conocer la identidad de la persona autora y, por consiguiente, deja de lado el hecho de que la revisión sea por lo menos doble ciega. Estas experiencias nos han hecho analizar si el sistema de revisión por pares que utiliza la revista es el adecuado. En la búsqueda de analizar otras opciones de revisión por pares encontramos la revisión abierta (en inglés open review). Este artículo repasa brevemente algunas ventajas y desventajas de este tipo de revisión y la experiencia de esta revista al implementarlo.
\end{abstract}

Palabras claves: revisión por especialistas; revisión abierta; arbitraje.

\section{ABSTRACT}

Mora-Campos, A. (2015). New ways of peer reviewing in journals: open review. PENSAR EN MOVIMIENTO: Revista de Ciencias del Ejercicio y la Salud, 13(1), 1-4. Technology has helped improve many aspects of the quality of publications: more visibility, greater access to sources of information and option to verify those sources, among others. This has meant that some journals have had to adjust their processes, especially peer reviewing. The blind mode is the method most commonly used by journals. In the case of our journal, we have traditionally used the double-blind style; however, we have noticed that, with advanced Internet, social networks, and media, it is 
easier for reviewers to obtain previews, preprints, primary research or previous articles that can help identify the author, thus making the double blind review difficult. These experiences have made us analyze whether the peer review system used by the journal is appropriate. Searching for other peer review options we found the open review. This paper briefly reviews some advantages and disadvantages of this type of review and the experience faced by this journal when implementing it.

Keywords: peer review; open review; arbitration.

Como editora de revistas he visto que el avance de la tecnología ha ayudado a mejorar muchos aspectos de la calidad de las publicaciones: más visibilidad, mayor acceso a las fuentes de información y opción de verificar fuentes, entre otros. Sin embargo, este avance nos ha exigido estar al tanto de nuevas corrientes de publicación. Por ejemplo, el uso de los llamados preprint (almacenar borradores previos de la investigación en repositorios institucionales o personales), los cuales son una tendencia, no solo debido a la tecnología, sino también por la inclinación actual al acceso abierto, es decir el acceso inmediato a las investigaciones antes de ser publicadas, ha implicado que algunas revistas tengamos que ajustar procesos, en especial en la de revisión por pares.

En las revistas científicas o académicas el proceso de revisión por pares, conocido también como arbitraje (en inglés: peer review) se utiliza para validar investigaciones, este fue introducido en el siglo XVII y revistas como Science y JAMA lo establecieron desde 1940; aunque especialistas indican que tiene muchos desaciertos, este sigue siendo el sistema más utilizado por las revistas académicas para poder evaluar los artículos científicos, y en especial la modalidad ciego, en este modo el autor o autora no sabe quiénes evaluaron su investigación, pero el cuerpo de revisores sí conocen la identidad del(os/as) autores(as) (Amsen, 2014).

En el caso de nuestra revista, tradicionalmente hemos utilizado el estilo de doble ciego, pero nos hemos dado cuenta que, con el avance del Internet, las redes sociales, o los medios de difusión es posible que muchos de los dictaminadores lleguen a obtener previos, preprint, investigaciones primarias o bien artículos anteriores que facilitan conocer la identidad de la persona autora $y$, por consiguiente, deja de lado el hecho de que la revisión sea por lo menos doble ciega. De tal manera que se entra aquí en un conflicto: el revisor continúa a sabiendas de conocer la identidad de la persona autora o debe optar por declarar que no realizará la revisión, por consiguiente se hace necesario buscar quien lo sustituya, con lo que esto implica, pues la mayoría de editores tenemos serios problemas con el reclutamiento de buenos revisores.

Estas experiencias nos han hecho analizar si el sistema de revisión por pares que utiliza la revista es el adecuado. Por ello, hace dos años me acerqué a Ana María Cetto, autoridad en el tema de las revista científicas y le comenté la idea de probar 
otras formas de revisión por pares, y ella me sugirió la lectura de algunos artículos sobre el tema y así valorar la opción más conveniente y pertinente para la revista.

En la búsqueda encontré varias opciones de revisión por pares, una de ellas fue la revisión abierta (en inglés open review). De la cual hay varios modelos, desde los que hacen la revisión a lo interno de la revista, y así, revisores y autores se conocen hasta aquellas que, como Launch of Biology Direct, publican junto al artículo los comentarios y nombres de los revisores, sistema que también usa British Medical Journal Open Launches (Amsen, 2014). Otra forma es publicarlo en un sitio web abierto a la discusión de la comunidad de la revista, la cual deja comentarios que pueden ser vistos públicamente (Rojas y Rivera, 2011).

Entre las ventajas que esta modalidad tiene están las siguientes según Amsen (2014) y Rojas y Riviera (2011) DeCoursey (2006):

- Los autores pueden conocer quiénes son los revisores.

- Se puede reducir el sesgo de los revisores.

- Aumenta la transparencia del proceso.

- Las críticas son más respetuosas y constructivas.

- Las revisiones realizadas pueden servir de ejemplo para revisores principiantes.

- Los revisores pueden tener el reconocimiento directo por el trabajo implicado en la realización de la revisión, a tal punto que muchas de las revistas han optado por incluir los DOI (digital objects identificador) para los reportes de los revisores.

- El hecho de indicar y advertirle al autor que va para un escrutinio abierto podría generar que, incluso, se cuiden de posibles plagios.

- De forma abierta se comparte la responsabilidad de la aceptación de un artículo entre el autor, el editor y los revisores.

Hay autores que refieren desventajas, tales como las indica DeCoursey (2006), con respecto a que algunos autores y revisores no les gusta el intercambio de ideas, que incluso hasta ganan "enemigos" por el proceso o, como lo que señala Badhu (2010), en relación con algunos comentarios sobre trabajos de autores de renombre que podrían estar sesgados.

También, nos hemos cuestionado si tenemos la cultura y la madurez para dejar que los autores conozcan quiénes son sus revisores, sin poner en duda la ética y profesionalismo de los participantes en el proceso.

Asimismo, decidimos hacer una prueba, la cual fue sumamente valiosa y enriquecedora: tanto el autor como los revisores, luego de la revisión pudieron compartir algunos otros criterios que enriquecieron la calidad del artículo. Mi percepción, y la del autor, fue que no solo el proceso es más constructivo, sino que se da un mayor compromiso de parte de los implicados, como lo indican Rojas 
y Rivera (2011). Si el proceso de la revisión de este artículo tiene un resultado final positivo será posible leer ese manuscrito y los comentarios de los revisores de forma pública en un futuro cercano.

La experiencia nos enseña que la innovación en este tipo de evaluaciones será un proceso constante, pero necesario y obligatorio. En la revista Pensar en Movimiento es probable que nos traslademos a ese modelo, no obstante, no es un proceso lineal, y, por ahora es optativo, siempre y cuando los revisores y autores estén de acuerdo en participar.

\section{Referencias}

Amsen, E. (2014). What is open peer review? Recuperado de http://blog.f1000research.com/2014/05/21/what-is-open-peer-review/

Badhu, B. (2010). Re: Open peer review system: advantages and disadvantages. Recuperado de http://www.bmj.com/rapid-response/2011/11/03/reopen-peerreview-system-advantages-and-disadvantages

Bornmann, L. \& Mungra, P. (May, 2011). Improving peer review in scholarly journals. European Science Editing, 37(2), 41-43. Recuperado de http://www.lutzbornmann.de/icons/bornmannEssay41-43.pdf

DeCoursey, T. (2006). Perspective: The pros and cons of open peer review. Nature, doi:10.1038/ nature04991 Recuperado de http://www.nature.com/nature/peerreview/debate/nature04991.html

Rojas, A. y Rivera, S. (2011). Guía de Buenas Prácticas para Revistas Académicas de Acceso Abierto. Paraguay: ONG Derechos Digitales. Recuperado de http://www.latindex.org/documentos/descargas/ManualBuenas Practica Revistas Academicas.pdf 